\title{
IL POSLANIE K VLADIMIRU MONOMACHU DEL VESCOVO DANIIL. ANCORA A PROPOSITO DELLA LETTERATURA DI DIREZIONE SPIRITUALE
}

\author{
FRANCESCA ROMOLI \\ Università di Pisa \\ francescaromoli@libero.it
}

\section{RIASSUNTO}

Oggetto del saggio è il Poslanie k Vladimiru Monomachu (Epistola a Vladimir Monomach) del vescovo Daniil († 1121). Attraverso un'analisi incentrata sulla funzione letteraria e pragmatica delle citazioni bibliche presenti nel testo, l'autrice ne dimostra l'appartenenza alla forma letteraria degli insegnamenti spirituali. Esamina quindi la motivazione liturgica e le circostanze storiche della sua genesi nel confronto con il Poslanie Vladimiru Monomachu o poste (Epistola a Vladimir Monomah sul digiuno) del metropolita Nikifor I († 1121), avanzando l'ipotesi che le due opere possano essere stata scritte nell'imminenza della Pasqua del 1114.

PAROLE CHIAVE: letteratura medievale slava orientale; insegnamenti spirituali; Poslanie $k$ Vladimiru Monomachu; vescovo Daniil († 1121); citazioni bibliche; retorica e pragmatica linguistica.

\section{BISHOP DANIIL'S EPISTLE TO VLADIMIR MONOMACH. ABOUT SPIRITUAL TEACHINGS}

\section{ABSTRACT}

The article focuses on the Epistle to Vladimir Monomach (Poslanie $k$ Vladimiru Monomachu) written by bishop Daniil († 1121). The author offers an analysis based on the literary and pragmatic function of biblical quotations within the text. Through it, she establishes that the Epistle belongs to the literary form of spiritual teachings. She then considers the liturgical and historical circumstances surrounding the genesis of the text by comparing it with the Epistle to Vladimir Monomach on fasting (Poslanie Vladimiru Monomachu o poste) written by mitropolit Nikifor I ( +1121$)$. The results of the comparison allow her to formulate the hypothesis that Daniil and Nikifor wrote their Epistles shortly before Easter in 1114.

KEY WORDS: Early Russian Literature; Spiritual Teachings; Poslanie k Vladimiru Monomachu; Bishop Daniil († 1121); Biblical Quotations; Rhetoric and Pragmatics.

\section{Premessa}

Oggetto di questo saggio è un'opera che è stata attribuita al vescovo di Jur'ev Daniil († 1121), il Poslanie $k$ Vladimiru Monomachu (Epistola a Vladimir Monomach). ${ }^{1}$ In questa sede esamineremo la forma letteraria di appartenenza, la motivazione liturgica e le circostanze storiche della genesi del testo nel confronto con un'altra opera rivolta allo stesso destinatario -il gran principe

\footnotetext{
1 Si adotta qui l'edizione curata da Ponyrko (1992: 56-57). Sulla tradizione manoscritta e a stampa del Poslanie k Vladimiru Monomachu si veda Ponyrko (1992: 55). Sull'attribuzione del testo al vescovo di Jur'ev Daniil e sulle altre ipotesi di attribuzione si confronti ancora Ponyrko (1992: 49-55), con la bibliografia ivi citata.
} 
Vladimir Monomach (1053-1125)- dal metropolita Nikifor I († 1121), il Poslanie Vladimiru Monomachu o poste (Epistola a Vladimir Monomah sul digiuno).

L'utilità di un'analisi di questo tipo sembra suggerita dal fatto che il Poslanie di Daniil è stato generalmente catalogato fra le epistole, ${ }^{2}$ ma presenta caratteristiche che non sono conformi ai tratti distintivi dell'epistolografia medievale slava orientale. ${ }^{3}$ Per struttura, funzione e funzionamento, il testo sembra piuttosto tipologicamente analogo al Poslanie o poste di Nikifor, con il quale potrebbe condividere, oltre al destinatario, anche la forma letteraria, la motivazione liturgica e le circostanze storiche della genesi.

Come si è potuto dimostrare in un recente studio, il Poslanie o poste può definirsi "epistola" solo nel senso originario, apostolico e neotestamentario del termine. Più precisamente, nel contesto della produzione scrittoria del medioevo slavo orientale, il testo rappresenta la forma letteraria degli insegnamenti (o istruzioni) spirituali. L'esistenza di questa forma letteraria, postulata sulla base del Poučenie $k$ duchovnomu čadu (Insegnamento al figlio spirituale) di Georgij Zarubskij (Romoli 2006), ha trovato conferma appunto nell'analisi del Poslanie o poste. Il lavoro di analisi, fondato sulla disamina della funzione letteraria e pragmatica delle citazioni bibliche, e dunque dell'organizzazione retorica del testo, ci ha inoltre permesso di definire con maggiore precisione le invariabili "di genere" ${ }^{4}$ della letteratura di direzione spirituale. $^{5}$

In particolare, sulla base dei dati che sono emersi dalla disamina del Poučenie $k$ duchoonomu čadu e del Poslanie o poste, si è ipotizzato che la letteratura di direzione spirituale si distingua dalle altre forme letterarie praticate in area slava orientale -con specifico riferimento alle forme affini delle epistole e dei sermoni- a livello sia strutturale, sia funzionale e di funzionamento. Per quanto attiene alla struttura del testo, gli insegnamenti spirituali mostrano un assetto generalmente bipartito in una parte di introduzione, l'exordium, che ha la funzione di praeparare, e in una parte di carattere parenetico, l'admonitio, che ha la funzione di commovere. Questo modello strutturale, testimoniato dal Poučenie $k$ duchovnomu čadu, può presentare anche una variante "complessa", come accade per il Poslanie o poste,

2 Si veda, a titolo di esempio, la raccolta di epistole curata da Ponyrko (1992), nella quale è incluso anche il Poslanie $k$ Vladimiru Momomachu (Ponyrko 1992: 49-58).

${ }^{3}$ Le caratteristiche dell'epistolografia slava orientale di epoca medievale sono descritte in Brogi Bercoff $(1983,1986)$.

${ }_{4}$ Sulla questione dell'applicabilità del principio di genere letterario alla descrizione della pratica scrittoria e alla classificazione della produzione slava ecclesiastica si possono consultare Seemann (1976), Picchio (1990), Naumow (1995), Živov (1995), Stantchev (1998), Levšun (2007), Levšun (2010).

${ }^{5}$ Il saggio dedicato al Poslanie o poste, e più in generale alla forma letteraria degli insegnamenti spirituali, è attualmente in fase di preparazione. 
con una parte teorica centrale, assimilabile a una tractatio thematis, interposta fra exordium e admonitio (Romoli 2006). ${ }^{6}$

Per quanto attiene alla funzione del testo, la letteratura di direzione spirituale aspira a tradurre la fede in un corretto agire (ortoprassi), perseguendo in ciò una finalità pragmatica. ${ }^{7}$ Attraverso gli strumenti dell'arte retorica, adottata come strategia di discorso, infatti, il padre spirituale attualizza la parola di Dio, ne adatta il messaggio all'interpretazione del presente e ne rinnovava il potenziale metamorfico nel tentativo di persuadere il destinatario a correggersi con l'azione (Romoli 2006). ${ }^{8}$ Il funzionamento degli insegnamenti spirituali, pertanto, appare governato dalle citazioni bibliche che percorrono il testo, la cui funzione si manifesta sia sul piano letterario, sia sul piano pragmatico.

Sul piano letterario, l'analisi del Poslanie o poste ha evidenziato che la funzione dell'elemento biblico si esplica a due livelli: la citazione, cioè, può svolgere un ruolo portante nella strutturazione semantica del discorso, assimilabile al ruolo della "chiave biblica tematica" nella definizione di Picchio (1977), e un ruolo di "ancoraggio" liturgico, legando il testo all'occasione liturgica che ne motiva la compilazione. Sul piano pragmatico, invece, si distinguono tre diversi livelli di uso a seconda del "grado di partecipazione" delle singole citazioni alla realizzazione dell'azione comunicativa nei suoi effetti perlocutori. La citazione, cioè, può presentare un monito o un'esortazione che l'autore rende attuali rivolgendoli direttamente al destinatario (livello attuativo), può contribuire alla costruzione dell'argomentazione (livello argomentativo), può concorrere all'esplicazione o all'esemplificazione del concetto che persuade all'azione (livello didattico-esplicativo e didatticoesemplificativo). ${ }^{9}$

Al fine di accertare l'appartenenza del Poslanie $k$ Vladimiru Monomachu alla forma letteraria degli insegnamenti spirituali, e per verificare ulteriormente la validità dell'ipotesi circa l'esistenza di una letteratura di direzione spirituale, si

6 Cf. supra, nota 5.

7 La finalità pragmatica è un tratto inerente alla letteratura slava ecclesiastica tout court (Seemann 1993, Garzaniti 1998, Garzaniti 2007), che appare particolarmente manifesto sia nella letteratura omiletica (Romoli 2009) sia nella letteratura di direzione spirituale (Romoli 2006; cf. supra, nota 5).

8 In questa prospettiva, l'insegnamento spirituale può essere considerato un macro-atto linguistico (Austin 1988, Conte 1977, van Dijk 1980). Sulla correlazione fra retorica e pragmatica linguistica, posto che "la retorica disciplina il discorso mirato all'azione, attraverso cui un parlante agisce su un ascoltatore per persuaderlo", Venier (2008: 28, 13) osserva che, da un punto di vista metodologico, "accostare retorica e pragmatica linguistica in sincronia significa fare della retorica una parte della pragmatica, quella che si occupa dell'azione persuasiva". Analogo è il discorso per i sermoni (Romoli 2009: 11-14, con la bibliografia ivi citata, e passim).

9 Cf. supra, nota 5. Sulla questione della funzione delle citazioni bibliche nella letteratura omiletica e nella letteratura di direzione spirituale si veda anche Garzaniti, Romoli (2013: 140144). 
rende dunque necessaria un'analisi che sia incentrata sulla componente biblica e retorica del testo. Per chiarire la motivazione liturgica e le circostanze storiche della genesi dell'opera, invece, sembra opportuno un confronto diretto con il Poslanie o poste.

\section{COMPONENTE BIBLICA E COMPONENTE RETORICA ${ }^{10}$}

\subsection{L'exordium}

Al pari del Poučenie $k$ duchovnomu čadu, il Poslanie $k$ Vladimiru Monomachu presenta una struttura bipartita in una parte di introduzione, l'exordium, e in una parte parenetica, l'admonitio. La parte introduttiva si apre con un'enumeratio degli attributi del destinatario del discorso, attraverso la quale l'autore ne esalta la virtù (encomium) -“Eletto nel ventre di tua madre da Dio onnipotente, fregiato del nome di sovrano e imperatore dal suo Figlio unigenito, il Signore nostro Gesù Cristo, custodito e fortificato nella prosperità della ragione e del potere di sovrano e imperatore dal suo santissimo e benevolo Spirito che dona la vita"- e ne definisce la posizione -"gran principe pio e amante di Cristo" (Ponyrko 1992: 56). ${ }^{11}$ Identifica poi se stesso e definisce la propria posizione, continuando tuttavia a intessere le lodi del destinatario, di cui ora celebra la devozione a Dio e il fervore nella preghiera -“io sono il vescovo D[aniil] [...] sono tale [...] grazie al tuo grande zelo per Dio e alla fervida fede [che nutri] in lui, e all'amore che profondi a noi miseri, e alla [tua] brama di preghiera" (Ponyrko 1992: 56). ${ }^{12}$

Attraverso la citazione di Mt 10, 41, le cui parole sono improntate allo schema argomentativo esortazione all'azione-promessa di ricompensa (protrope), Daniil asserisce l'esistenza di un rapporto di proporzionalità diretta fra l'atto del donare e l'atto del ricevere: "Disse Cristo Salvatore nostro: 'Chi accoglie un profeta nel nome del profeta avrà la ricompensa del profeta, chi accoglie un giusto nel nome del giusto avrà la ricompensa del giusto'" (Ponyrko 1992: 56). ${ }^{13}$ A questa prima citazione se ne aggiunge una seconda, Mt 25, 40, con la quale l'autore rende esplicita la motivazione che giustifica e legittima l'affermazione precedente (aetiologia), enunciando il concetto della presenza di

\footnotetext{
${ }^{10}$ Per la definizione delle figure retoriche si farà riferimento a Lausberg (1998), Lanham (1968), Marchese (19844).

11 (Богомъ вседержителемъ нареченному изъ чрева матере своея, единочадымъ его Сыномъ, Господемь нашим Исус Христомъ, почтеному господьскымъ и царьскымъ саномъ, и пресвятымъ и благимъ и животворящимъ Духомъ его зблюдаему и усиляему на прђдспъние господьских же и царьскых разумъ и державы, благочестивому и христолюбивому великому князю [...]).

12 (азъ же Д епископъ [...] се же [...] твоего же ради еже къ Богу потщания велия и еже к нему въры теплыя и до насъ, худыхъ, простретья любве, еже хотьниа молитвы).

13 (Рекше Спасу нашему Христу: «Приемляи пророка во имя пророче мызду пророчю приемлеть, приемляи праведника въ имя праведниче мьзду праведничю приемлеть»).
} 
Dio nei più umili: "poiché ciò che faceste [a uno solo] di questi miei fratelli più piccoli, lo faceste a me" (Ponyrko 1992: 56). ${ }^{14}$

Le parole del vangelo pongono a fondamento del Poslanie i principi della buona condotta, dell'equità e dell'umiltà. Richiamando l'attenzione del gran principe sul rapporto di proporzionalità esistente fra gli atti del donare e del ricevere, la citazione di Mt 10, 41 allude all'idea della giustizia divina e della conformità del giudizio finale alla virtù o al vizio di ognuno, svelando i due livelli di lettura e di interpretazione del discorso, i cui moniti acquistano significato nella duplice prospettiva della vita terrena e della vita eterna, e nella previsione assoluta della salvezza o della dannazione. In questo senso, la citazione di Mt 10, 41 assolve la funzione di chiave biblica tematica del Poslanie. Il modello logico su cui è strutturata (protrope) le conferisce inoltre forza attuativa. La citazione di Mt 25, 40, che invece è integrata nel discorso a scopo argomentativo, ne accresce l'efficacia motivando l'esortazione all'equità attraverso il riferimento alla virtù dell'umiltà.

\subsection{L'admonitio}

L'admonitio si apre con un nuovo encomium che Daniil rivolge al destinatario per celebrare il dono divino che egli possiede di ricercare in ogni cosa un vantaggio per la propria salute e per la propria salvezza. In questo modo, l'autore enuncia la premessa da cui muoverà il discorso in questa parte e la finalità del discorso stesso: “È proprio della tua anima devota, e anima divina, e [della tua] ragione divina cercare in tutte le cose e da tutte [trarre] vantaggio per la propria salvezza e salute. È un dono di Dio avere un tale buon senso nel proprio cuore" (Ponyrko 1992: 56). ${ }^{15}$ Espone poi la tesi centrale, ribadendo, ora con valore di avvertimento (paraenesis), l'idea della proporzionalità fra l'atto del ricevere e l'atto del donare attraverso la citazione di Lc 12, 48, che richiama e rinnova le parole di Mt 10, 41: "In verità, infatti, bisogna preoccuparsi di queste cose, secondo la parola del Salvatore: 'A chi fu dato molto, molto sarà chiesto'" (Ponyrko 1992: 56). ${ }^{16}$ In questo contesto, la citazione di Lc 12, 48 ha scopo argomentativo, poiché è funzionale all'enunciazione della tesi del Poslanie, ma mostra anche una finalità attuativa, poiché l'autore rivolge il monito in essa racchiuso direttamente destinatario.

Per poter rispondere adeguatamente alle richieste che gli saranno avanzate in sede di giudizio, il gran principe, che ha ricevuto molto, dovrà mostrarsi ugualmente generoso nel donare. Daniil si adopera allora per persuaderlo della necessità di adottare una condotta ispirata alle parole di Lc

\footnotetext{
14 («еже понеже створисте от менших си братии моеи, и мнъ створисте»).

${ }^{15}$ (Се же есть твоеа благочестивыа душа, и божественыя душа, и божественыя разумъ, еже въ всъхъ и от всъхъ искати ползы своему спасению иже и здравию. Се же есть даръ Божии, еже есть таковъ разумъ имъти въ сердци своемъ).

16 (Поистине бо и достоино еже о сицевыхъ пещися, по Спасову слову: «Емуже дасться много, и много въстяжется от него»).
} 
12,48 , attualizzandone il messaggio a scopo attuativo e didattico-esplicativo. Lo esorta a profondersi per condurre a Dio i suoi sudditi, promettendogli di ricevere in cambio vantaggi commisurati alle opere che avrà compiuto (protrope):

Per questo motivo è necessaria una guida alla tua devozione, affinché nel tuo regno tu conduca molti a Dio, poiché ti chiami figlio di Dio ed effondi la grazia e l'immagine di Dio. E allora [Dio] ti darà per quanto avrà ricevuto, e ti preserverai nella ragione (Ponyrko 1992: 56).17

Presenta poi tre exempla concreti ai quali il destinatario potrà rifarsi, ricordando prima il suo bisavo, il gran principe Vladimir Svjatoslavič (960ca1015), di cui esalta lo zelo in Dio, l'attitudine al bene e l'opera di evangelizzazione dello Stato, poi il progenitore Jaroslav Vladimirovič (983/9861054), di cui celebra l'inclinazione verso il prossimo, e infine il padre Vsevolod Jaroslavič (1030-1093), di cui evoca la mitezza, la clemenza, il timore di Dio e la giustizia.

Attraverso un'enumeratio delle virtù che ha appena elencato, ora Daniil sprona il destinatario a esercitarsi in quelle stesse virtù per affrancarsi dal peccato e ottenere il perdono di Dio. L'efficacia correttiva dell'esortazione si fonda su una previsione di ricompensa (protrope) che l'autore enuncia esplicitando l'antithesis "vita terrena - vita eterna", con la promessa per il gran principe di ricevere serenità e lunga vita su questa terra per poi ricongiungersi a Dio nei cieli:

E di quello ricordando la conversione dal male al bene e lo zelo dell'azione apostolica, di questo la grande sollecitudine verso gli uomini, e del tuo santo padre la correzione virtuosa, ed esercitatoti in tutte queste [virtù], e ricevuti subito da Dio la correzione e il perdono, vivrai senza inquietudini per lunghi anni nel regno presente, e del regno futuro, ineffabile ed eterno, sarai compartecipe con Dio, che è più in alto dell'intelletto e del pensiero dell'uomo (Ponyrko 1992: 56).18

Nel rinnovato tentativo di indurre il gran principe alla correzione, Daniil si rivolge a lui con una supplica (deesis) che offre la citazione di Mt 6, 14, esortandolo alla misericordia che realizza la parola di Dio: "Prego per questo la tua signoria, affinché tu sia misericordioso verso chi ha peccato contro di te, realizzando la parola del Salvatore che disse: 'Se rimetterete agli uomini i loro

17 (Сего ради требъ и много смотрение твоему благочестию, да и многихъ управиши къ Богови въ своемъ царствъ, понеже сынъ Божии наречеся по благодати и образъ Божии поносиши. Да и подасть ти, еже приять, да и зблюдеши, в твоемъ бо есть разумъ).

18 (Да оного поминая еже от здобы на добро совращение и потщание апостодьскаго управления, сего же - еже о людъхъ попечение много, святаго же ти отца - еже добродътелное исправление, да съми всими потщався исправлении и приимше от Бога прошение твое въскоръ, и безмятежно и долгольтно поживеши в нынъшнемъ царствии и будущаго и неизреченнаго и бесконечнаго царства съобъщникъ съ Богомъ будеши, иже есть выше ума и смысла человека). 
peccati, il Padre vostro celeste rimetterà a voi i vostri peccati'" (Ponyrko 1992: 56). ${ }^{19} \mathrm{Il}$ riferimento a Mt 6, 14, che presenta in forma di protrope il concetto della reciprocità del perdono, si lega, esplicitandola, alla citazione di Mt 10, 41 dell'exordium, e conferisce potere attuativo all'invito per il destinatario ad agire secondo le scritture. Il messaggio di Mt 6, 14 è enfatizzato dalla successiva citazione di Mt 18, 22, integrata nel discorso come raccomandazione (testamentum), e dunque a scopo attuativo: “Disse il Signore: 'Settanta volte sette perdonateli, rimettete al fratello i suoi peccati'" (Ponyrko 1992: 56-57). ${ }^{20}$

L'ultima esortazione, che rinnova l'invito rivolto al gran principe a dimostrarsi misericordioso concedendo il perdono, ripropone il modello logico esortazione all'azione-promessa di ricompensa (protrope), subordinando alla messa in atto del precetto evangelico la promessa per il destinatario di ottenere la perfezione divina: "Come in molte cose, anche in questo si manifesti la tua misericordia, 'sii perfetto -disse il Salvatore- come è perfetto il Padre vostro celeste'" (Ponyrko 1992: 57). ${ }^{21}$ Il Poslanie si conclude con una benedizione (eulogia) attraverso la quale Daniil invoca sul gran principe la misericordia e la protezione di Dio e della Madre di Dio, chiedendo che il destinatario sia preservato dal male e possa ottenere la vittoria sui nemici in questa vita, per poi accedere alla vita eterna: “Dio, per la sua misericordia e per le preghiere della santa Madre di Dio, ti preservi nel tuo regno da ogni male e ti conceda vittorie sui nemici [e] nel secolo futuro la vita eterna, per la grazia e l'amore per l'uomo del suo Figlio unigenito, nei secoli, amen" (Ponyrko 1992: 57). ${ }^{22}$ In chiusura, dunque, Daniil si richiama all'antithesis "vita terrena - vita eterna", nella cui duplice prospettiva, posta nell'exordium dalla citazione di Mt 10, 41, deve essere letto l'intero discorso.

\section{MOTIVAZIONE LITURGICA E “MARCATORI LITURGICI"}

L'analisi del Poslanie $k$ Vladimiru Monomachu, di cui abbiamo indagato la forma, la funzione e il funzionamento sulla base della componente biblica e della componente retorica, sembra provare l'attinenza del testo con la letteratura di direzione spirituale, mostrandone la conformità ai parametri formali, funzionali e di funzionamento che regolano il Poučenie $k$ duchovnomu čadu e il Poslanie o poste, e che si è ipotizzato contraddistinguano la forma letteraria degli insegnamenti spirituali.

\footnotetext{
19 (Молю же о семъ твое державное царство, да и милостивъ будеши съгръшающимъ к тобъ, исполняа Спасово слово, еже рече: «Аще оставите человекомъ съгьршения их, и Отець вашь небесныи оставить вамъ съгьршения ваша»).

20 (Рекшу Господу: «70 седмерицею пращаите ихъ, оставдяите брату съгрбшения его»).

${ }^{21}$ (Да якоже въ многихъ, такоже и о семъ явится человеколюбие твое «да будеши съврьшенъ - Спасу рекшу - якоже Отецъ вашь небесныи свершенъ есть»).

22 (Богъ милостию своею и молитвами святыя Богородица съблюдет тебе въ царствии твоемъ от всякого зла и на противныя побъды даруеть, в будущемъ въци жизнь въчную, благодатию и человеколюбием единороднаго Сына твоего (sic!) в въкы, аминь).
} 
Nel Poslanie o poste, tuttavia, la funzione letteraria dell'elemento biblico non si esaurisce nel ruolo di chiave tematica svolto da una citazione biblica, ma si manifesta anche a livello liturgico. Il testo, infatti, accoglie due citazioni tratte dal vangelo di Matteo (Mt 6, 12; 6, 14) che appartengono alle letture della settimana di astensione dai latticini. ${ }^{23}$ La loro presenza conferma che il Poslanie fu rivolto al gran principe in occasione dell'inizio del Grande digiuno pasquale, come l'autore afferma a più riprese. Nikifor esordisce infatti con queste parole: "Benedetto è Dio [...] che ci ha concesso di giungere a questi rispettabilissimi giorni del santo digiuno" (Ponyrko 1992: 66), ${ }^{24}$ per poi definire la forma del discorso -“Nella forma di un'istruzione, secondo il regolamento ecclesiastico" (Ponyrko 1992: 67)-, ${ }^{25}$ e precisarne la motivazione -“E per questo noi osammo, poiché il regolamento e la regola ecclesiastica [prescrivono] in questo periodo [di] dire qualcosa di utile anche ai principi" (Ponyrko 1992: 70). ${ }^{26}$

Il Poslanie o poste offre dunque la prova che la stesura di un insegnamento spirituale rivolto da un alto prelato (in questo caso il metropolita stesso) a un'alta carica statale (in questo caso il gran principe) era prevista e richiesta, in determinate occasioni liturgiche, dal regolamento ecclesiastico. A livello letterario, l'appartenenza di due delle citazioni bibliche presenti nel testo alla liturgia della stessa occasione per la quale il testo era stato compilato, ovvero l'inizio della Quaresima, mostra che la motivazione liturgica di un insegnamento e la sua attinenza con una determinata liturgia potevano essere formalizzate attraverso l'inserimento al suo interno di citazioni tratte dalle letture previste per quella stessa liturgia.

Le citazioni bibliche presenti nel Poslanie di Daniil, allora, potrebbero essere eloquenti al fine di stabilire se il vescovo di Jur'ev lo rivolse al gran principe in occasione di una ricorrenza liturgica particolare. Di fatto, fra il Poslanie o poste e il Poslanie di Daniil, in aggiunta alla coincidenza di forma letteraria e di destinatario di cui si è detto -entrambe le opere sono insegnamenti spirituali ed entrambe sono rivolte a Vladimir Monomach- si osserva una coincidenza che investe il piano delle citazioni bibliche e che potrebbe essere rilevante per definire la motivazione liturgica del Poslanie di Daniil. Nel testo, infatti, ricorre una delle citazioni tratte dal vangelo di Matteo, Mt 6, 14, che nel Poslanie o poste conferma la motivazione liturgica a cui Nikifor fa esplicito riferimento, ribadendo il legame del testo con la liturgia della

\footnotetext{
${ }^{23}$ La lettura di Mt 6, 12; 6, 14 era prevista, nell'ordine, il sabato e la domenica di astensione dai latticini (Garzaniti 2001: 487). La settimana di astensione dai latticini è l'ultima settimana del secondo ciclo liturgico (da Pentecoste a Quaresima), e precede l'inizio della Quaresima (terzo ciclo liturgico).

24 (Благословенъ Богъ [...] сподобивъ ны доити въ пръчестыя дни сиа святаго поста).

25 (Въ образъ же наказаниа по церковному уставу).

26 (И сего ради дрьзнухом мы, яко уставъ есть церковнныи и правило - въ время се и къ княземь глаголати что полезное).
} 
settimana di astensione dai latticini. ${ }^{27}$ Nel Poslanie di Daniil, allora, la citazione di Mt 6, 14 potrebbe essere il "marcatore" che formalizza il legame dell'opera con quella stessa liturgia, lasciando ipotizzare che, in ottemperanza al regolamento ecclesiastico, a cui Nikifor si richiama, sia il metropolita di Kiev sia gli altri vescovi della Rus' fossero tenuti a rivolgere un insegnamento scritto al gran principe in vista dell'inizio del Grande digiuno pasquale.

\section{Circostanze StORiche}

L'ipotesi che Daniil abbia rivolto il suo Poslanie a Vladimir Monomach nella stessa occasione liturgica in cui Nikifor indirizzò al medesimo destinatario il Poslanie o poste non implica, tuttavia, che i due testi si collochino nello stesso frangente storico, ovvero che siano stati scritti in occasione dell'inizio della Quaresima di uno stesso anno, ma, d'altra parte, non esclude neanche questa ipotesi. Per accertare se, oltre alla forma letteraria, al destinatario e, come pare verosimile, alla motivazione liturgica, il Poslanie di Daniil condivida con il Poslanie o poste anche le circostanze storiche della genesi, occorre raccogliere e comparare i dati noti utili alla datazione dei due testi.

Il Poslanie o poste non è datato, né presenta elementi che ne consentano una datazione certa. L'epoca della sua stesura, pertanto, è stata determinata indirettamente, fissando il termine ante quem al 1113, anno dell'ascesa al trono kieviano di Vladimir Monomach, e il termine post quem al 1121, anno della morte di Nikifor. In considerazione della motivazione liturgica dell'opera, questo lasso temporale è stato poi ulteriormente delimitato comparando le date di intronamento del gran principe e la data della Pasqua del 1113. Risultando la prima (20 aprile) posteriore alla seconda (6 aprile), si è concluso che il termine ante quem dovesse essere posticipato al Grande digiuno che precedette la Pasqua del 1114 (29 marzo), e si è ipotizzata una datazione prossima all'anno 1117 (Čičurov 1990: 141). ${ }^{28}$ I punti di contatto esistenti fra il Poslanie o poste e il Poučenie $v$ nedelju syropustnuju (Insegnamento nella domenica di astensione dai latticini) dello stesso autore -dalla coincidenza della chiave biblica tematica alla convergenza dei "marcatori" e della motivazione liturgica dei due testi ${ }^{2}{ }^{2}$ convince, tuttavia, della vicinanza anche cronologica di queste opere. Secondo una recente datazione, la compilazione del Poučenie v nedelju syropustnuju risalirebbe alla Quaresima del 1113 e sarebbe stata motivata dai disordini che precedettero l'ascesa al trono di Vladimir Monomach (Poljanskij 2000: 273, Poljanskij 2006: 194 nota 8). Nikifor potrebbe allora aver redatto il Poslanie o poste l'anno immediatamente successivo, in occasione del digiuno pasquale del 1114.

\footnotetext{
${ }^{27}$ Cf. supra e nota 23.

${ }^{28}$ Il termini ante quem e post quem fissati da Čičurov sono condivisi e riproposti da Kazaku, Starostina (2003: 232) e Barankova (2005: 23).

${ }^{29}$ Cf. supra, nota 5. Sul Poučenie v nedelju syropustnuju si veda anche Romoli (2009: 42-92).
} 
Al pari del Poslanie o poste, il Poslanie di Daniil non reca date né si presta a una datazione precisa. La sua stesura deve necessariamente collocarsi fra l'anno dell'insediamento di Daniil sulla cattedra di Jur'ev (1114) e l'anno della morte del presule (1121). Tichomirov ha ipotizzato che l'opera sia stata suscitata dalla repressione degli oppositori di Vladimir Monomach successiva al suo intronamento e possa rappresentare una sorta di replica al Poslanie o poste, scritta in difesa del gran principe. Lo studioso colloca dunque la compilazione del Poslanie di Daniil (e indirettamente anche quella del Poslanie o poste) nel 1113 (Tichomirov 1960: 455). ${ }^{30}$ Come si è visto, il Poslanie o poste non può essere datato al 1113, ma potrebbe piuttosto risalire al 1114. La datazione al 1113 mal si adatta anche al Poslanie di Daniil, per due ragioni. In primo luogo, secondo gli annali, Daniil occupò la cattedra di Jur'ev il 6 gennaio del 1113 nello stile di marzo (Lavr.let. 1846: 127), data che nello stile moderno corrisponde al 6 gennaio del 1114. Quando Vladimir Monomach si insediò a Kiev, il 20 aprile del 1113, dunque, Daniil non era ancora vescovo di Jur'ev. In secondo luogo, la motivazione liturgica (quaresimale) del Poslanie da noi ipotizzata escluderebbe in ogni caso la datazione del testo al 1113, visto che l'intronamento di Vladimir Monomach avvenne dopo la Pasqua del 1113.

Il legame che Tichomirov intuisce fra il Poslanie o poste e il Poslanie di Daniil, che sul piano della forma letteraria e del destinatario è palese, e sul piano della motivazione liturgica ci sembra almeno verosimile, lascerebbe allora ipotizzare che anche Daniil, come Nikifor, abbia rivolto il suo insegnamento a Vladimir Monomach in occasione del Grande digiuno del 1114, forse memore degli eventi che l'anno precedente avevano scosso la capitale della Rus', e comunque in ottemperanza del regolamento ecclesiastico. Restano forse ancora celate, o sono andate per sempre perdute, opere di analoga fattura che nella stessa epoca anche altri vescovi della Rus' devono aver rivolto ai principi attenendosi alle prescrizioni del regolamento ecclesiastico.

\section{CONCLUSIONI}

L'analisi del Poslanie Daniil e il confronto con il Poslanie o poste hanno prodotto una serie di risultati che permettono di formulare alcune conclusioni. Le caratteristiche strutturali, la funzione e il funzionamento del testo sembrano provare l'appartenenza del Poslanie alla forma letteraria degli insegnamenti spirituali, recando un'ulteriore prova dell'esistenza di questa forma come autonoma e distinta sia dalla letteratura omiletica sia dall'epistolografia. Il testo mostra una struttura bipartita in exordium e admonitio, tipologicamente analoga alla variante strutturale semplice testimoniata dal Poučenie $k$ duchovnomu čadu. Sul piano della funzione, il Poslanie persegue lo scopo pragmatico di persuadere il destinatario all'azione attraverso l'attualizzazione in forma retoricamente

${ }^{30}$ La stessa datazione è riproposta in Tvorogov (1987). 
marcata della parola di Dio e del suo potenziale correttivo. L'elemento biblico rappresenta pertanto il cardine del funzionamento del testo. Come si è mostrato, la citazione di Mt 10, 41 (exordium) ha il ruolo di chiave biblica tematica, svelando i due livelli di lettura e di interpretazione del discorso (funzione letteraria). Dal punto di vista pragmatico, tutte le citazioni bibliche presenti nel testo concorrono a realizzare l'azione comunicativa nei suoi effetti perlocutori, ognuna in misura diversa sulla base di tre livelli di uso (che non si escludono): Mt 6, 14, Mt 10, 41, Mt 18, 22 e Lc 12, 48 sono integrati nel discorso a scopo attuativo, poiché presentano esortazioni che l'autore rivolge direttamente al destinatario; Mt 25, 40 e Lc 12, 48 concorrono alla formulazione dell'argomentazione finalizzata a persuadere; Lc 12, 48 è usato anche a scopo didattico-esplicativo (funzione pragmatica).

Dal confronto del Poslanie di Daniil con il Poslanie o poste, inoltre, sono emersi alcuni dati eloquenti sulla cui base è stata ipotizzata la presenza nel testo (e in testi dello stesso tipo) di uno o più "marcatori liturgici". Nel Poslanie o poste, infatti, la motivazione liturgica dell'insegnamento, che, per ammissione dello stesso autore, fu scritto in occasione dell'inizio del Grande digiuno, trova conferma nella presenza nel testo di due citazioni (Mt 6, 12; 6, 14) tratte dalle letture previste per la liturgia della settimana di astensione dai latticini. Si è allora ipotizzato che questo meccanismo di "ancoraggio" alla liturgia caratterizzi in generale la forma letteraria degli insegnamenti spirituali, ivi compreso il Poslanie di Daniil. Da un esame volto ad accertare l'attinenza liturgica dei passi biblici presenti al suo interno è emerso che la citazione di Mt 6, 14 appartiene alle letture della domenica di astensione dai latticini. Tenendo conto della disposizione del regolamento ecclesiastico a cui Nikifor fa riferimento nel Poslanie o poste, secondo la quale nell'imminenza del periodo quaresimale le alte cariche ecclesiastiche erano tenute a rivolgere un discorso ai principi, si è allora ipotizzato che, al pari di Nikifor, anche Daniil abbia rivolto il suo insegnamento a Vladimir Monomach in occasione dell'inizio del digiuno pasquale. Per quanto attiene la funzione delle citazioni bibliche, la presenza nel Poslanie o poste e nel Poslanie di Daniil di citazioni che formalizzano il legame del testo con una determinata liturgia permette allora di ipotizzare che nella forma degli insegnamenti spirituali la funzione letteraria dell'elemento biblico si esplichi non solo sul piano della strutturazione semantica del discorso, nel ruolo di chiave biblica tematica, ma anche sul piano della motivazione liturgica del testo, nel ruolo di "marcatore liturgico".

Per ultimo, le coincidenze che si osservano fra il Poslanie di Daniil e il Poslanie o poste ci hanno indotto a indagare le circostanze storiche della genesi dei due testi. In questo ambito, tenendo conto del fatto che le due opere non sono datate, e comparando i dati noti utili alla loro datazione, si è avanzata l'ipotesi che entrambi gli insegnamenti possano essere stati scritti nell'imminenza della Pasqua del 1114, e forse essere stati suscitati dai disordini che avevano accompagnato l'ascesa di Vladimir Monomach al trono kieviano. 
In conclusione, ci sembra che i risultati emersi dall'analisi del Poslanie di Daniil incoraggino a proseguire la ricerca nell'ambito della letteratura di direzione spirituale, per verificare ulteriormente $\mathrm{i}$ tratti distintivi di questa forma letteraria, ampliare lo spettro delle invariabili che la contraddistinguono e accertare il grado di variabilità ammesso al suo interno. Nell'ambito particolare degli insegnamenti spirituali rivolti da un alto prelato a un alta carica statale -opere che potrebbero rappresentare una precisa tipologia all'interno della letteratura di direzione spirituale-, sarebbe inoltre auspicabile individuare e studiare, fra le testimonianze a noi note, altri testi che potrebbero essere stati redatti in ottemperanza alle prescrizioni per il periodo quaresimale previste dal regolamento ecclesiastico. Eventuali riscontri positivi in questo settore permetterebbero di meglio comprendere e valutare il significato di opere di cui molto spesso si percepisce il carattere genericamente edificatorio, senza tuttavia riuscire a cogliere quella che nelle intenzioni dell'autore e nella contingenza storica doveva essere la loro destinazione reale.

\section{BIBLIOGRAFIA}

Austin, J. L. (1988), Come fare cose con le parole. Le William James lectures tenute alla Harvard university nel 1955, Penco, C., Sbisà, M. (eds.). Ed. or. Austin, J.L. (1962), How to do Things with Words. The William James Lectures delivered at Harvard University in 1955, Oxford, Clarendon Press.

Barankova, G. S. (2005), Čista molitva tvoja. Poučenie i poslanija drevnerusskim knjaz'jam kievskogo mitropolita Nikifora, Moskva, ICHTIOS.

BROGI BERCOFF, G. (1983), “L'epistolografia russa antica e il suo rapporto con quella bizantina", en Mondo slavo e cultura italiana. Contributi italiani al IX Congresso internazionale degli slavisti (Kiev 1983), Kresalkova, J. (ed.), Roma, Il Veltro Editrice, 55-77.

BROGi BERCOFF, G. (1986), "Critères d'étude de l'épistolographie russe médiévale”, en Studia slavica medievalia et humanistica Riccardo Picchio dicata, Colucci, M., Dell'Agata, G., Goldblatt, H. (eds.), vol. 1, Roma, Edizioni dell'Ateneo, 55-77.

ČIČUROV, I. S. (1990), Političeskaja ideologija srednevekov'ja. Vizantija i Rus'. K XVIII Meždunarodnomu kongressu vizantinistov, Moskva, Nauka.

CONTE, M.-E. (ed.) (1977), La linguistica testuale, Milano, Feltrinelli.

VAN DIJK, T. A. (1980), Testo e contesto. Semantica e pragmatica del discorso, Bologna, Il Mulino (ed.or. van Dijk, T.A. [1977], Text and Context. Explorations in the Semantics and Pragmatics of Discourse, London, Longman).

GARZANITI, M. (1998), “L'agiografia slavo-ecclesiastica nel contesto della liturgia bizantina. Sacra Scrittura e liturgia nella composizione letteraria della Vita di Paraskeva", en Contributi italiani al XII Congresso internazionale degli slavisti (Cracovia 26 agosto-3 settembre 1998), Esvan, F. (ed.), Napoli, [C.I.S.C.S.F.], 1998, 87-129.

GARZANITI, M. (2001), Die altslavische Version der Evangelien. Forschungsgeschichte und zeitgenössische Forschung, Köln, Weimar, Wien, Böhlau. 
GARZANITI, M. (2007), "Biblejskie citaty v literature Slavia Orthodoxa", Trudy Otdela drevnerusskoj literatury, 58, 28-40.

GARZANITI, M., ROMOLI, F. (2013), "Le funzioni delle citazioni bibliche nella letteratura della Slavia ortodossa", en Contributi italiani al XV Congresso Internazionale degli Slavisti (Minsk, 20-27 settembre 2013), Garzaniti, M., Alberti, A., Perotto, M., Sulpasso, B. (eds.), Firenze, Firenze University Press, 121-156.

KAZAKU, M., STARostina, I. P. (2003), “Poslanie Nikifora, mitropolita kievskogo, k velikomu knjazju Vladimiru Monmachu o poste", en Pis'mennye pamjatniki istorii Drevnej Rusi. Letopisi. Povesti. Choždenija. Poučenija. Žitija. Poslanija. Annotirovannyj katalog-spravočnik, Ščapov, Ja.N. (ed.), Sankt Peterburg, Russko-Baltijskij informacionnyj centr "BLIZ", 232-234.

LANHAM, R. A. (1968), A Handlist of Rhetorical Terms, Berkeley, Los Angeles, Universiry of California Press.

LAUSBERG, H. (1998), Handbook of Literary Rhetoric. A Foundation of Literary Study, Leiden, Boston, Köln, Brill (ed.or. Lausberg, H. [1960], Handbuch der literarischen Rhetorik. Eine Grundlegung der Literaturwissenschaft, 2 vv., München, Max Hueber).

LAVR.LET. (1846), Lavrent'evskaja i Troickaja letopisi, Sankt Peterburg, Tipografija Ėduarda Praca (Polne sobranie russkich letopisej, I/1-2).

LEVŠUN, L. V. (2007), “Zakon ritoričeskoj imitacii: $k$ voprosu o principach funkcionirovanija sistemy metodov, stilej, žanrov v srednevekovoj vostočnoslavjanskoj knižnosti", Drevnjaja Rus', 30 (2007/4), 25-34.

LEVŠUN, L. V. (2010), “'Koncepcii stilja' v vostočnoslavjanskoj knižnosti XI-XVII v.”, Drevnjaja Rus', 40 (2010/2), 16-26.

MARCHESE, A. (19844), Dizionario di retorica e stilistica, Milano, Mondadori.

NAUMOW, A. (1995), "Srednevekovaja literatura i bogosluženie”, Ricerche Slavistiche, 42, 49-59.

PicchiO, R. (1977), "The Function of Biblical Thematic Clues in the Literary Code of 'Slavia Orthodoxa'”, Slavica Hierosolymitana, 1, 1-31 (reprint en Picchio, R. [1991], Letteratura della Slavia ortodossa [IX-XVIII sec.], Bari, Dedalo, 363-403).

PICCHIO, R. (1990), “Povest' e Slovo. Osservazioni sul rapporto fra narrativa e omiletica nella tradizione scrittoria dell'antica Rus'", Europa Orientalis, 9, 23-36.

POLJANSKIJ, S. M. (2000), "Religiozno-filosofskaja problematika v "Poslanii o poste" mitropolita Nikifora", en Filosofskie i bogoslovskie idei v pamjatnikach drevnerusskoj mysli, Gromov, M.N., Mil'kov, V.V. (eds.), Sankt Peterburg-Moskva, Nauka, 270283.

POLJANSKIJ, S. M. (2006), Tvorenija mitropolita Nikifora, Moskva, Nauka.

PONYRKO, N. V. (1992), Épisoljarnoe nasledie Drevnej Rusi XI-XIII. Issledovanija, teksty, perevody, Sankt Peterburg, Nauka.

Romoli, F. (2006), "Il Poučenie k duchovnomu čadu di Georgij Zarubskij e la letteratura di direzione spirituale", Russica Romana, 13, 15-31.

Romoli, F. (2009), Predicatori nelle terre slavo-orientali (XI-XIII sec.). Retorica e strategie comunicative, Firenze, Firenze University Press.

SEEMANN, K.-D. (1976), Die Altrussische Wallfahrtsliteratur. Theorie und Geschichte eines literarischen genres, München, Fink.

SEEMANN, K.-D. (1993), "Priemy allegoričeskoj eksegezy v literature kievskoj Rusi", Trudy Otdela drevnerusskoj literatury, 48, 105-120. 
STANTCHEV, K. (1998), “Žanr, stil' i modus v srednevekovoj literature pravoslavnych slavjan. Metodologičeskie i terminologičeskie problemy", en Contributi italiani al XII Congresso internazionale degli slavisti (Cracovia 26 agosto-3 settembre 1998), Esvan, F. (ed.), Napoli, [C.I.S.C.S.F.], 1998, 25-60.

TICHOMIROV, M. N. (1960), "Maloizvestnye pamjatniki", Trudy Otdela dreonerusskoj literatury, 16, 452-456.

Tvorogov, O. V. (1987), “Daniil, episkop Jur'evskij”, en Slovar' knižnikov i knižnosti Dreonej Rusi, Lichačev, D.S. (ed.), vol. 1, Leningrad, Nauka, 109.

VENIER, F. (2008), Il potere del discorso. Retorica e pragmatica linguistica, Roma, Carocci.

ŽIVOV, V. M. (1995), “Osobennosti recepcii vizantijskoj kul'tury v Drevnej Rusi", Ricerche Slavistiche, 42, 3-48 (reprint en Živov, V.M. [2002], Razyskanija v oblasti istorii i predystorii russkoj kul'tury, Moskva, Jazyki slavjanskoj kul'tury, 73-115). 